\title{
Energy and Throughput Analysis of Hierarchical Routing Protocol (LEACH) for Wireless Sensor Network
}

\author{
Rajesh Patel \\ M.Tech \\ Institute of Technology, \\ Nirma University, \\ Ahmedabad, Gujarat, India.
}

\author{
Sunil Pariyani \\ M.Tech \\ Institute of Technology, \\ Nirma University, \\ Ahmedabad, Gujarat, India.
}

\author{
Vijay Ukani \\ Assistant Professor - CSE \\ Institute of Technology, \\ Nirma University, \\ Ahmedabad, Gujarat, India.
}

\begin{abstract}
Wireless sensor networks (WSNs) have gained increasing attention from both the research community and actual users. The efficient utilization of energy source in a sensor node is very important criteria to prolong the life time of wireless sensor network. Wireless sensor networks have explored to many new protocols specifically designed for sensor networks where energy consideration is very crucial. Most of importance, given to hierarchical routing protocols based on clustering has better scalability. As sensor nodes are generally battery-powered devices, the critical aspects to face concern how to reduce the energy consumption of nodes, so that the network lifetime can be extended to reasonable times. There are several energy efficient hierarchical routing protocols among this LEACH is famous protocol, we have simulated LEACH in NS2 and analyzed performance of LEACH in terms of energy, throughput and lifetime.
\end{abstract}

Keywords: LEACH, Hierarchical Routing Algorithms, clustering, wireless sensor networks.

\section{INTRODUCTION}

A Wireless Sensor Networks (WSN) is a set of hundreds or thousands of micro sensor nodes that have capabilities of sensing, establishing wireless communication between each other and doing computational and processing operations. The important requirements [1] of a WSN are: (1) Use of a large number of sensors (2) Attachment of stationary sensors (3) Low energy consumption (4) Self-organization capability (5) Collaborative signal processing, and (6) Querying ability. Some of the important application domains of WSNs are listed below.

- Military environment,

- Disaster management

- Habitat monitoring

- Medical and health care,

- Industrial fields, Home networks

- Biological, radiological, nuclear, and explosive material etc.

Sensor nodes are equipped with small, often irreplaceable batteries with limited power capacities. They can be deployed manually or be randomly dropped. They are self configuring, containing one or more sensors, with embedded wireless communications and data processing components and a limited energy source. The use of wireless sensor networks is increasing day by day but the problem of energy constraints prevails as there is limited battery life. In order to save energy dissipation caused by communication in wireless sensor networks, it is necessary to schedule the state of the nodes, changing the transmission range between the sensing nodes, use of efficient routing and data routing methods and avoiding the handling of unwanted data. In general, routing in WSNs [2] can be divided into flat, hierarchical, and location based routing depending on the network structure.

Hierarchical Routing is the well-known technique with special advantages related to scalability and efficient communication. LEACH, PEGASIS, TEEN [3] and APTEEN use this technique for routing. In hierarchical architecture, higher energy nodes can be used to process and send the information, while low-energy nodes can be used to perform the sensing in the proximity of the target. LocationBased Routing Protocols like MECN [4] sensor nodes are addressed by means of their locations. The distance between neighboring nodes can be estimated on the basis of incoming signal strengths. Relative coordinates of neighboring nodes can be obtained by exchanging such information between neighbors. The Low-Energy Adaptive Clustering Hierarchy $(\mathrm{LEACH})$ is a cluster based routing protocol. In this paper section 2 will introduce the LEACH routing protocol in detail, Section 3 will cover the simulation of LEACH protocol and the section 4 shows the simulation analysis by varying the percentage of cluster heads in the network in each simulation of LEACH protocol. Performance is analyzed in terms of lifetime, energy dissipation and throughput of the network and Section 5 concludes this paper.

\section{REVIEW OF LEACH PROTOCOL}

Low-Energy Adaptive Clustering Hierarchy (LEACH) protocol for sensor networks is proposed by W. R. Heinzelman et.al [5] which minimizes energy dissipation in sensor networks. It is very famous hierarchical routing algorithms for sensor networks which make clusters of the sensor nodes based on the received signal strength shown in figure 1 . The $5 \%$ of the total number of nodes becomes the cluster head which act as router to the sink. Energy 
consumption is less as transmission will only be done by cluster head.

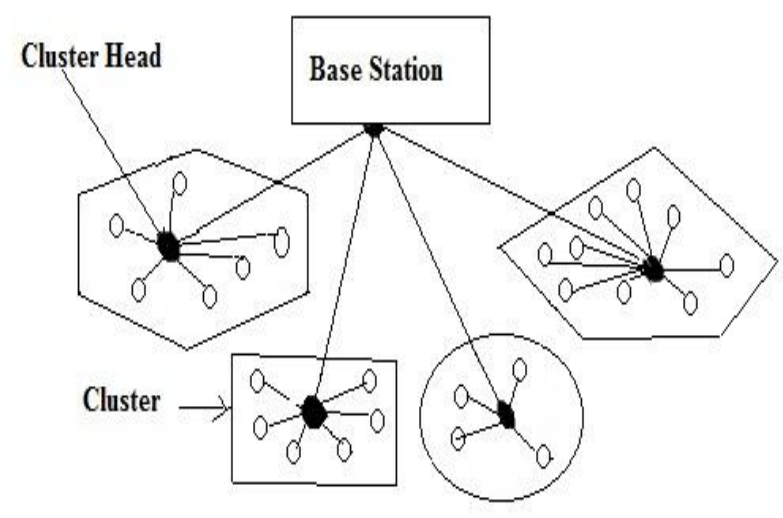

Fig. 1: cluster based mechanism of LEACH in WSN

Data fusion and aggregation are local to the cluster. Cluster heads change randomly over time to balance the energy dissipation of nodes. The node chooses a random number between 0 and 1 . The node becomes a cluster head for the current round if the number is less than the following threshold:

$$
T(n)=\left\{\begin{array}{cl}
\frac{p}{1-p *\left(r \bmod \frac{1}{p}\right)} & \text { if } n \in G \\
0 & \text { otheriwse }
\end{array}\right.
$$

Where $p$ is the desired percentage of cluster heads (e.g. 0.05), $\mathrm{r}$ is = the current round, and $\mathrm{G}$ is the set of nodes that have not been cluster heads in the last $1 / p$ rounds.

Set-up phase: During this phase, each node decides whether or not to become a cluster head $(\mathrm{CH})$ for the current round. This decision is based on choosing a random number between 0 and 1 , if number is less than a threshold $\mathrm{T}(\mathrm{n})$, the node become a cluster head for the current round.

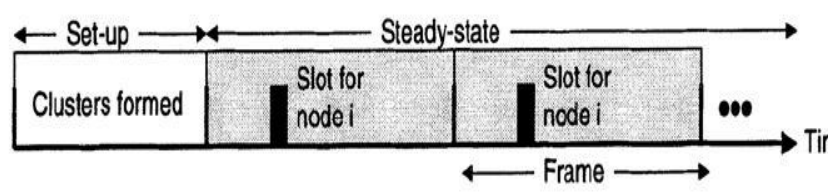

Fig. 2: Time Line operation of LEACH [6]

The cluster head node sets up a TDMA schedule and transmits this schedule to all the nodes in its cluster, completing the setup phase which is then followed by a steady-state operation.
Steady-state phase: The steady-state [4] operation is broken into frames, where nodes send their data to the cluster head at most once per frame during their allocated slot shown in figure 2. It assumes nodes always have data to send, they send it during their allocated transmission time to the cluster head. This transmission uses a minimal amount of energy (chosen based on the received strength of the cluster-head advertisement). The radio of each non-cluster-head node can

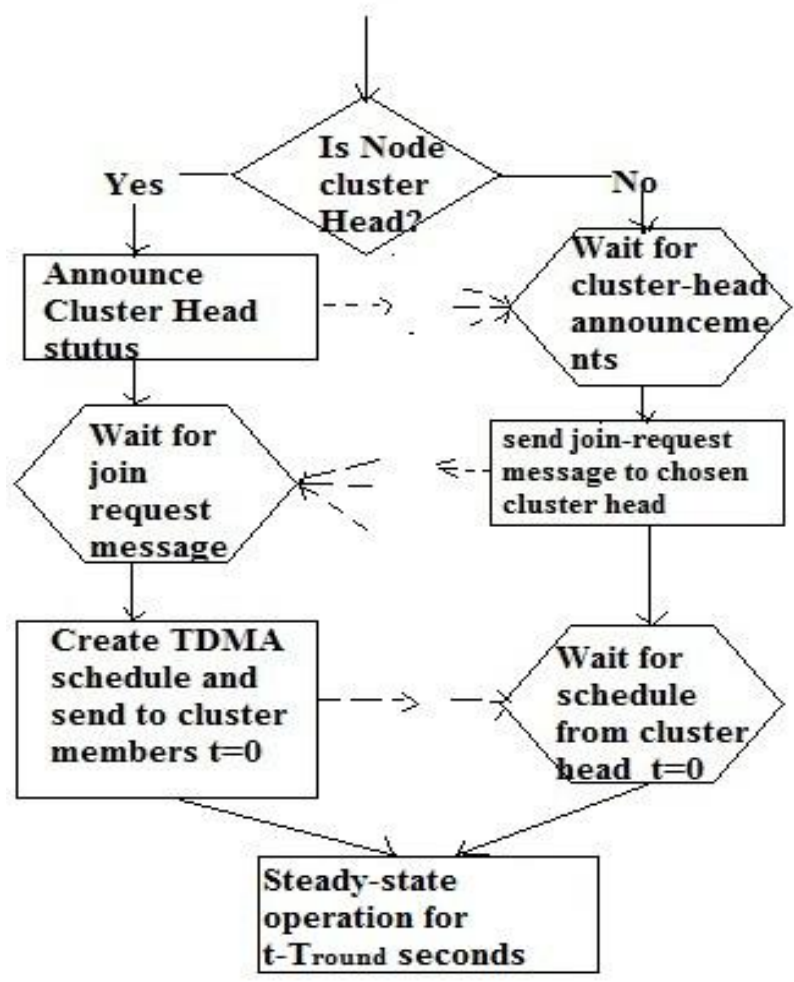

Fig. 3: Flow chart of the Set-up phase of the LEACH protocol

be turned off until the node's allocated transmission time, thus minimizing energy dissipation in these nodes. The cluster-head node must keep its receiver on to receive all the data from the nodes in the cluster. When all the data has been received, the cluster head node performs signal processing functions to compress the data into a single signal. For example, if the data are audio or seismic signals, the clusterhead node can beamform the individual signals to generate a composite signal. This composite signal is sent to the base station. Since the base station is far away, this is a highenergy transmission.

\section{SIMULATION OF LEACH PROTOCOL}

To simulate the LEACH protocol, MIT's NS2 extension for LEACH simulator [7] [8] is used. The models were used for channel propagation and energy dissipation is as described below: 


\subsection{Channel Propagation Model}

In the wireless channel, the electromagnetic wave propagation can be modeled as falling off as a power law function of the distance between the transmitter and receiver. The free space model which considered direct line-of-sight and two-ray ground propagation model which considered ground reflected signal also, were considered depending upon the distance between transmitter and receiver. If the distance is greater than $\mathrm{d}_{\text {crossover }}$, two-ray ground propagation model is used. The crossover is defined as follows.

$$
\mathrm{d}_{\text {crossover }}=\frac{4 * \pi * \sqrt{L} * \mathrm{~h}_{\mathrm{r}} * \mathrm{~h}_{\mathrm{t}}}{\lambda}
$$

Where, $\mathrm{L} \geq 1$ is system loss factor. Here equation $2, \mathrm{~h}_{\mathrm{r}}$ is the height of the receiving antenna, $h_{t}$ is the height of the transmitting antenna and $\lambda$ is the wavelength of the carrier signal. Now transmit power is attenuated based on following formula:

$\mathrm{P}_{\mathrm{r}}(\mathrm{d})= \begin{cases}\frac{\mathrm{P}_{\mathrm{t}} * \mathrm{G}_{\mathrm{t}} * \mathrm{G}_{\mathrm{r}} * \lambda^{2}}{(4 * \pi * \mathrm{~d})^{2} * \mathrm{~L}} & \text { if } \mathrm{d}<\mathrm{d}_{\text {crossover }} \\ \frac{\mathrm{P}_{\mathrm{t}} * \mathrm{G}_{\mathrm{t}} * \mathrm{G}_{\mathrm{r}} * \mathrm{~h}_{\mathrm{t}}{ }^{2} * \mathrm{~h}_{\mathrm{r}}{ }^{2}}{\mathrm{~d}^{4}} & \text { if } \mathrm{d} \geq \mathrm{d}_{\text {crossover }}\end{cases}$

Where, $P_{r}$ is the received power at distance $d, P_{t}$ is transmitted power, $G_{t}$ is gain of the transmitting antenna and $G_{r}$ is gain of the receiving antenna.

\subsection{Radio Energy dissipation Model}

We assumed a simple model for the radio hardware energy dissipation where the transmitter dissipates energy to run the radio electronics and the power amplifier, and the receiver dissipates energy to run the radio electronics as shown in figure 4. Using this radio model, to transmit k-bit message at distance $\mathrm{d}$ the radio expends:

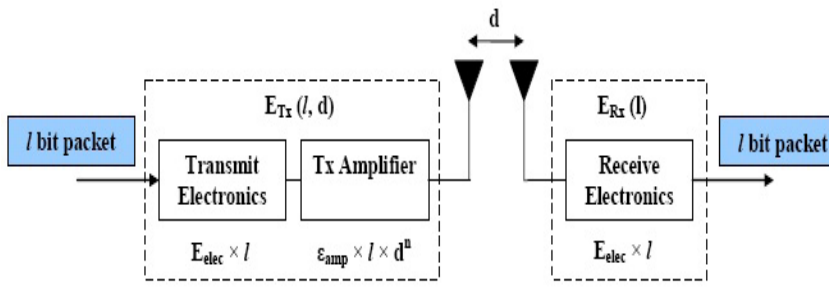

Fig. 4: Radio energy dissipation model [8]

$E_{T x}(k, d)=E_{T x-e l e c}(k)+E_{T x-a m p}(k, d)$

$E_{T x}(k, d)=E_{\text {elec }} * k+\epsilon_{\text {amp }} * k * d^{2}$

and to receive this message, the radio expends:

$$
\begin{aligned}
& E_{R x}(k)=E_{R x-e l e c}(k) \\
& E_{R x}(k)=E_{\text {ele }} * k
\end{aligned}
$$

\section{SIMULATION ANALYSIS 4.1 Simulation Parameters}

We simulated the MIT's LEACH cluster based routing protocol using NS 2.27[9]. By varying the parameter Percentage of cluster head in the MIT's LEACH configuration file, analyzed the performance of the network in terms of lifetime of the sensor network, throughput achieved and total energy consumption by the sensor network. For the experiment, percentage of cluster heads 2, $3,4,5,6,7$ and 8 of the total sensor nodes are taken. For the simulation experiment, following parameter was used:

$T_{x}$ antenna gain $G_{t}=R x$ antenna gain $G_{r}=1$ Antenna Height $h_{t}=1.5 \mathrm{~m}$,

No system loss $(\mathrm{L}=1), 914 \mathrm{MHz}$ radios and $\lambda=\frac{3 * 10^{8}}{914 * 10^{6}}=0.328 \mathrm{~m}$.

\begin{tabular}{|c|c|c|}
\hline $\begin{array}{l}\text { No. } \\
\text { Item }\end{array}$ & $\begin{array}{l}\text { No. Item } \\
\text { Description } \\
\text { Specification }\end{array}$ & $\begin{array}{l}\text { No. Item Description } \\
\text { Specification }\end{array}$ \\
\hline 1 & Simulation Area & $1000 \times 1000$ \\
\hline 2 & No. of nodes & 100 \\
\hline 3 & $\begin{array}{l}\text { Transmitter } \\
\text { amplifier } \\
\text { Energy } \\
\text { dissipation } \\
\text { (a) } \mathrm{E}_{\mathrm{fs} \text { amp }} \\
\text { (b) } \mathrm{E}_{\text {two ray amp }}\end{array}$ & $\begin{array}{l}10 \mathrm{pJ} / \mathrm{bit} / \mathrm{m}^{2} \\
0.0013 \mathrm{~nJ} / \mathrm{bit} / \mathrm{m}^{4}\end{array}$ \\
\hline 4 & Radio bit rate- $\mathrm{Rb}$ & $1 \mathrm{mbps}$ \\
\hline 5 & Channel Type & $\begin{array}{l}\text { Channel/wireless } \\
\text { channel }\end{array}$ \\
\hline 6 & $\begin{array}{l}\text { Radio } \\
\text { propagation } \\
\text { model }\end{array}$ & Two ray ground \\
\hline 7 & Simulation time & $600 \mathrm{~s}$ \\
\hline 8 & Antennae model & Antenna/omniantenna \\
\hline 9 & Energy model & Battery \\
\hline 10 & $\begin{array}{l}\text { Interface queue } \\
\text { type }\end{array}$ & $\begin{array}{l}\text { Queue/Drop tail/ } \\
\text { priqueue }\end{array}$ \\
\hline 11 & Link layer type & LL \\
\hline 12 & $\begin{array}{l}\text { Communication } \\
\text { model }\end{array}$ & Bi-direction \\
\hline 13 & Min packet in ifq & 30 \\
\hline
\end{tabular}

Base station location was $(50,175)$

Table 1. Simulation Parameters

\subsection{Simulation Results}

The Results of the simulation are shown in the Table 2, which shows the Lifetime, Energy and Throughput of the different no. of clusters or cluster heads in the sensor network, here $5 \%$ clusters heads of total network nodes are more energy efficient and also throughput is good as compare to others. Figure 5, 6 and 7 shows the simulation graphs for percentage of cluster heads verses lifetime, throughput and average energy dissipation respectively 
Table 2. Simulation Results

\begin{tabular}{|c|c|c|c|}
\hline $\begin{array}{c}\text { No. of } \\
\text { Clusters/ } \\
\% \text { Cluster } \\
\text { Head }\end{array}$ & $\begin{array}{c}\text { Lifetime } \\
(\mathbf{s})\end{array}$ & Throughput & $\begin{array}{c}\text { Energy } \\
\text { Consumpti } \\
\text { on } \\
(\mathbf{J})\end{array}$ \\
\hline 2 & 361.00 & 30097 & 395.00 \\
\hline 3 & 285.09 & 32279 & 425.00 \\
\hline 4 & 464.10 & 35897 & 338.00 \\
\hline 5 & 542.30 & 52127 & 292.03 \\
\hline 6 & 464.00 & 42041 & 348.64 \\
\hline 7 & 353.53 & 29426 & 323.99 \\
\hline 8 & 181.39 & 8301 & 294.49 \\
\hline
\end{tabular}

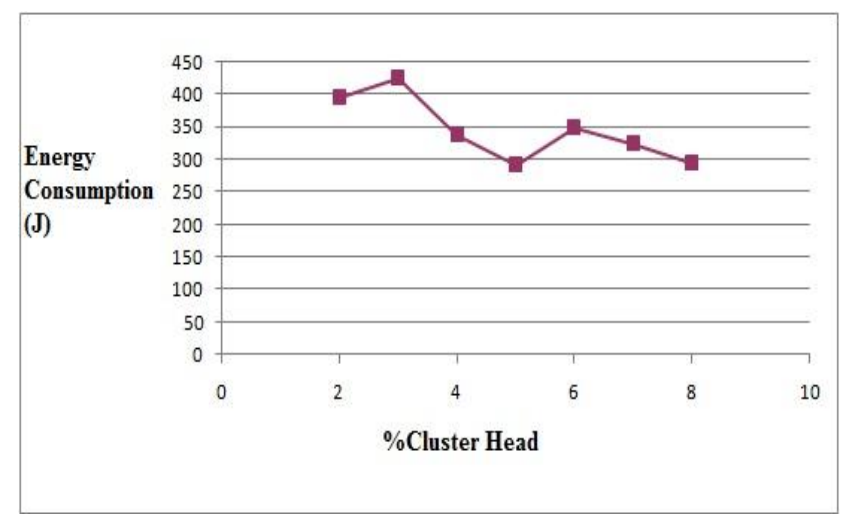

Fig. 5: No. of clusters Vs Energy dissipation of the network.

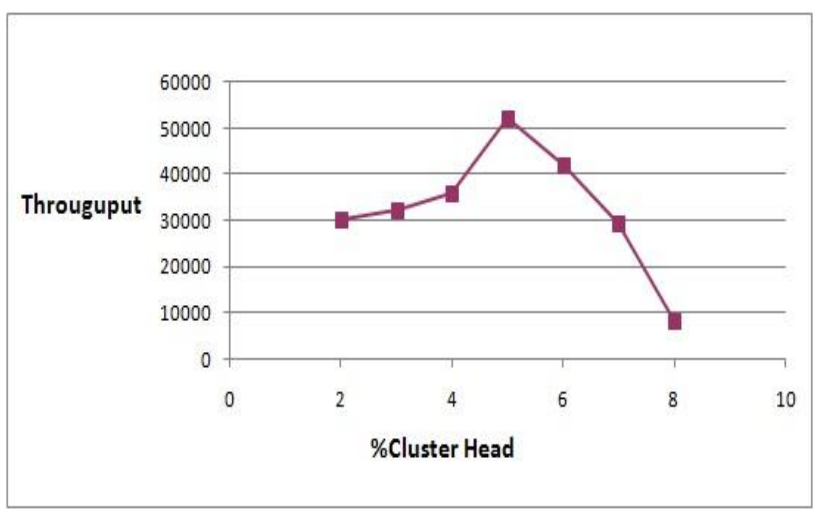

Fig. 6: No. of clusters Vs Throughput of the network.

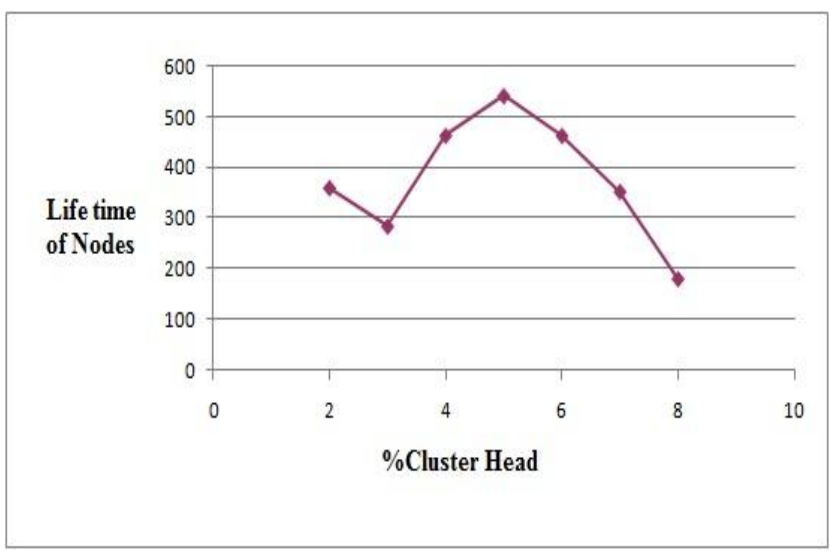

Fig. 7: No. of clusters Vs Lifetime of the network.

\subsection{LEACH Assumption/Limitations}

Although LEACH is able to increase the network lifetime, there are still a number of issues about the assumptions used in this protocol. LEACH [9] assumes a homogeneous distribution of sensor nodes in the given area. This scenario is not very realistic.

LEACH assumes that all nodes can transmit with enough power to reach the BS if needed and that each node has computational power to support different MAC protocols. Therefore, it is not applicable to networks deployed in large regions.

It also assumes that nodes always have data to send and nodes located close to each other have correlated data. It is not obvious how the number of predetermined Cluster Heads $[\mathrm{CH}(\mathrm{p})]$ is going to be uniformly distributed throughout the network. Therefore, there is a possibility that the elected $\mathrm{CHs}$ will be concentrated in one part of the network. Hence, some nodes will not have any $\mathrm{CHs}$ in their vicinity.

\section{CONCLUSION}

The Hierarchical routing protocol LEACH is energy efficient for the sensor network. By varying the different no. of clusters heads/clusters in the network the performance of network changed in terms of lifetime, throughput and average energy dissipation. From the above results we concluded that if the clusters in network or cluster heads in network are below or above 5 percentage of the total no. of nodes the performance of the network is degraded in terms of energy, throughput and lifetime so, when the no. of clusters heads are 5 percentage of the sensor nodes then the performance is good.

\section{ACKNOWLEDGEMENT}

We thank to Mr. Gaurang Raval to provide us important material to simulate this protocol. 


\section{REFERENCES}

[1] Nitaigour P. Mahalik, "Sensor Networks and Configuration: Fundamentals, Standards, Platforms, and Applications", Chapter 1, 7 \& Spinger, 2007.

[2] Jamal N. Al-Karaki, Ahmed E. Kamal. "Routing Techniques In WirelessSensor Networks: A Survey", IEEE Wireless Communications,December 2004.

[3] A. Manjeshwar and D. Agrawal, "TEEN: a routing protocol for enhanced efficiency in wireless sensor networks", In Proceedings 15th International Parallel and Distributed Processing Symposium, pp. 2009-2015. 23-27 April 2001.

[4] V. Rodoplu, T.H. Ming, "Minimum energy mobile wireless networks", IEEE Journal of Selected Areas in Communications, Vol. 17, No. 8, pp. 1333-1344, 1999.

[5] W. R. Heinzelman, A. Chandrakasan, and H. Balakrishnan, "Energy Efficient Communication Protocol for Wireless Micro Sensor Networks", Proceedings of IEEE HICSS, Jan 2000.
[6] P.T.V. Bhuvaneswari and V.Vaidehi, "Enhancement techniques incorporated in LEACH- A Survey", Indian Journal of Science and TechnologyVol.2 No 5 (May 2009)

[7] Massachusetts Institute of Technology, Cambridge, "The MIT uAMPSns Code Extensions, Version 1.0" August, 2000 .

[8] Sanjeev Saini, Ram Sewak Singh \& V.K.Gupta "Analysis of Energy Efficient Routing Protocols in Wireless Sensor Networks Communication", IJCSC Vol. 1, No. 1, January-June 2010, pp. 113-118.

[9] Liu Lintao Yang Ping, " Routing Protocols Simulation Based on NS2", Ship Electronic Engineering [J], 2008.4 Vo.1 28 No. 4:132-134

[10] Lan Tien Nguyen, Xavier Defago, Razvan Beuran, Yoichi Shinoda, "An Energy Efficient Routing Scheme for Mobile Wireless Sensor Networks", IEEE ISWCS 2008. 